\title{
EFEITO DO MANEJO E DE VARIÁVEIS BIOCLIMÁTICAS SOBRE A TAXA DE GESTAÇÃO EM VACAS RECEPTORAS DE EMBRIÕES
}

\author{
Eliane Vianna da Costa e Silva, ${ }^{1}$ Kamyla Ayumi Katayama, ${ }^{2}$ Gustavo Guerino Macedo, ${ }^{2}$ Paola Moretti \\ Rueda, ${ }^{2}$ Urbano Gomes Pinto de Abreu ${ }^{3}$ e Carmem Estefânia Serra Neto Zúccari ${ }^{4}$ \\ 1. Professora doutora, Laboratório de Reprodução Animal, Faculdade de Medicina Veterinária e Zootecnia, UFMS. E-mail: licsilva@nin.ufms.br \\ 2. Programa de Mestrado em Ciência Animal, Faculdade de Medicina Veterinária e Zootecnia, UFMS \\ 3. Embrapa Pantanal, Corumbá, MS \\ 4. Departamento de Zootecnia, Faculdade de Medicina Veterinária e Zootecnia, UFMS. \\ Grupo de Estudos em Reprodução Animal do Estado de Mato Grosso do Sul, GERA-MS / CNPq.
}

\section{RESUMO}

Para verificar o efeito do manejo e do ambiente sobre a taxa de gestação de receptoras bovinas cruzadas zebu x europeu $(\mathrm{n}=94)$ de embriões Nelore registraram-se o comportamento e variáveis clínicas e climáticas durante o protocolo de sincronização de cio e no dia da inovulação. Foi determinada a concentração de cortisol plasmático $(\mathrm{n}=36)$ no D0, D9 e D16 do protocolo e de progesterona (P4) no dia da inovulação. Não houve diferença significativa entre fêmeas gestantes (GEST) e não gestantes (nGEST) quanto ao estágio de maturação e classificação do embrião, pelagem, comportamento ou entre embriões descongelados e fresco. A concentração média de cortisol variou significativamente entre D0 e D16 $(\mathrm{P}>0,001)$. A P4 do D16 foi menor nas nGEST $(\mathrm{P}<0,01)$. O índice de temperatura de globo e umidade foi elevado, indicando que os animais poderiam estar sob estresse por calor. As fêmeas GEST apresentaram menor temperatura de pele $(\mathrm{P}<0,01)$. A análise de regressão mostrou aumento da probabilidade de prenhez de cerca de $25 \%$ entre o início e o final da tarde $(\mathrm{P}=0,08)$. A menor concentração plasmática de progesterona no momento da inovulação e a maior temperatura da pele demonstraram o desconforto térmico e influenciaram negativamente a fertilidade das receptoras de embriões. O estresse térmico por calor afetou a probabilidade de prenhez de vacas receptoras de embriões sob condições tropicais.

PALAVRAS-CHAVES: Cortisol, estresse por calor, gado de corte, manejo, taxa de gestação.

\section{ABSTRACT}

\section{EFFECT OF HANDLING AND ENVIRONMENTAL VARIABLES ON THE PREGNANCY RATE OF RECIPIENT COWS}

This work aimed to verify the effects of the handling and thermal environment on the pregnancy rate of embryo-recipient zebu $x$ European cows $(\mathrm{n}=94)$ as well as the behavioral and clinical aspects at the moment of embryo transfer. Cortisol was analyzed by plasmatic samplers collected on D0, D9 and D16 of the hormonal treatment for estrous synchronization; blood was also sampled to analyze progesterone levels at the moment of embryo transfer. There was no difference $(p>0.05)$ between pregnant and not pregnant females for maturation stage and embryo classification, coat, and reactivity between fresh and frozen embryos. The cortisol concentrations decreased $(p>0.001)$ between D0 and D16, and the concentration of progesterone was similar in function of the pregnancy stage. Nevertheless progesterone in D16 for non pregnant cows was lower than for pregnant ones in the same day. Moreover, pregnant cows showed lower skin temperature. The values of Black Globe-Humidity Index were at very high limits, indicating that all animals could be under heat stress. The regression analysis indicated a tendency $(\mathrm{p}=0.08)$ to increase the pregnancy probability of $25 \%$ between the beginning and end of the afternoon. Thus, low plasmatic concentration of progesterone and high skin temperature represents a factor of thermal discomfort at the moment of the embryo receiving, influencing the pregnancy rate of recipient cows. As observed, heat stress decreased the probability of the pregnancy of recipient cows in tropical environment.

KEYSWORDS: Beef cattle, cortisol, handling, heat stress, pregnancy rate. 


\section{INTRODUÇÃO}

O manejo reprodutivo em programas de inseminação artificial demanda contato constante do homem com os animais, justamente em momentos em que o estresse pode ter efeitos negativos sobre a eficiência reprodutiva. $\mathrm{O}$ comportamento de cio pode ser diminuído ou suprimido sob diferentes estímulos ambientais, tais como tipo de piso, inibição social, calor intenso, barulho etc. (ORIUHELA, 2000; LANDAETA-HERNÁNDEZ et al., 2002). Em programas de transferência de embriões pouco se tem estudado a respeito do efeito do ambiente sobre as taxas de gestação de receptoras.

Em ambientes tropicais, a alta temperatura do ar, associada à umidade e radiação elevadas, reduz a eficiência da perda de calor e, com isso, incrementa o estresse do animal (SILVA et al., 2006), limitando o desenvolvimento, a produção e a reprodução (LANDAETA-HERNANDEZ et al., 2002; VASCONCELOS et al., 2006; TORRES-JÚNIOR et al., 2008). A capacidade do animal de se ajustar fisiologicamente ao estresse por calor, em função de altas temperaturas e umidade relativa do ar, tem sido avaliada fisiologicamente por alterações da temperatura retal e da frequência respiratória, explicando a tentativa do animal em perder o calor absorvido (AZEVEDO et al., 2005; MAIA et al., 2005; MORAIS et al., 2008). Um ambiente é considerado confortável quando o animal está em equilíbrio térmico com ele, ou seja, em que o calor produzido pelo metabolismo é perdido para o meio ambiente sem prejuízo da homeostase do animal. Alguns índices têm sido usados para medir o conforto ou o desconforto dos animais em relação às condições ambientais, entre eles o índice de temperatura e de umidade (ITU), calculado com base na combinação de temperatura e de umidade, e o índice de temperatura de globo e umidade (ITGU), calculado com base na umidade e na radiação recebida pelo animal (SILVA, 2000).

Vacas holandesas expostas ao estresse por calor por pelo menos um dia de temperatura máxima $>29^{\circ} \mathrm{C}$ antes da inseminação artificial obtiveram proporção de gestação menor $(\mathrm{P}<0,001)$ que vacas não expostas a este fator (CHEBEL et al., 2004). VASCONCELOS et al. (2006) observaram que, em vacas Holstein receptoras de embriões, o aumento da temperatura retal diminuiu a taxa de prenhez no dia 25 pós-inovulação $(\mathrm{P}<0,05)$ e aumentou a perda embrionária $(\mathrm{P}<0,10)$. TORRES-JÚNIOR et al. (2008) observaram efeito deletério do estresse por calor sobre a dinâmica folicular ovariana e sobre a competência do ovócito de vacas Gir.

A concentração plasmática de progesterona é um fator importante para obtenção de êxito na transferência de embriões. Receptoras de embriões que apresentavam concentração plasmática de P4 entre 2,0 e 5,0 ng/mL no momento da inovulação obtiveram taxa de prenhez superior àquelas que apresentavam concentração abaixo de 2,0 ng/mL ou acima de 5,0 ng/mL (REMSEN \& ROUSSEL, 1982; NIEMANN et al., 1985).

Portanto, se forem usadas técnicas de manejo que propiciem melhores concentrações plasmáticas adequadas de progesterona, potencialmente poderá haver melhora na taxa de prenhez.

As consequências do clima tropical no desempenho reprodutivo de bovinos têm sido pouco estudadas no Brasil e, em uma situação de manejo específico e constante como observado em programas de transferência de embriões, os fatores climáticos podem ter impacto maior. Este trabalho teve por finalidade testar a hipótese de que fatores bioclimáticos podem afetar a prenhez de receptoras bovinas de embriões.

\section{MATERIAL E MÉTODOS}

O estudo foi realizado na Fazenda Sete Estrelas Embriões, localizada no município de Terenos, Mato Grosso do Sul (20²0'0” S, 55'2'52" W, altitude 301 metros), nos meses de fevereiro e março de 2004. Registraram-se dados de três lotes de receptoras mestiças provenientes de cruzamento industrial (Europeu $\mathrm{x}$ Nelore) e com a mesma condição corporal: lote1 $(n=38)$, lote $2(n=26)$ e lote $3(n=30)$, totalizando 94 fêmeas, sendo que, deste total, 25 foram inovuladas com embriões congelados e 69 com embriões a fresco oriundos de doadoras da raça Nelore. As doadoras eram vacas adultas com idade média de 5,9 $\pm 4,1$ anos, já tendo sido submetidas a pelo menos duas coletas, mantidas em ótimas condições nutricionais, com escore corporal médio 7.0 (WILTBANK, 1983).

Submeteram-se as receptoras a um protocolo hormonal de sincronização de cio com as doadoras até a inovulação do embrião. No dia zero (D0), foi 
inserido um dispositivo intravaginal progesterona (Cidr ${ }^{\circledR}$, Pfizer) e aplicados $2 \mathrm{~mL}$ de benzoato de estradiol (Estrogin ${ }^{\circledR}$, Farmavet). No quinto dia (D5) aplicaram-se $2 \mathrm{~mL} \mathrm{PGF}_{2} \alpha$ (Ciosin ${ }^{\circledR}$, Coopers $)+2 \mathrm{~mL}$ de eCG (Novormon ${ }^{\circledR}$, Tecnopec). No oitavo dia (D8), foi retirado o $\mathrm{Cidr}^{\circledR}$, no nono dia (D9) aplicaram-se $2 \mathrm{~mL}$ de Estrogin ${ }^{\circledR}$ e no décimo sexto dia (D16) as receptoras receberam os embriões.

No dia da inovulação (D16), procedeu-se à avaliação das receptoras por meio de exame ginecológico, via ultrassom, pela manhã. Feito o exame, elas aguardavam em um piquete e à tarde eram levadas para um corredor de acesso à sala de transferência, calculando-se, em minutos:

- o tempo de manejo das receptoras (t manejo), considerando o intervalo entre o horário de chegada no curral de manhã até o horário de entrada no corredor;

- o tempo de espera ( $t$ espera) definido como o intervalo de tempo do momento de chegada do piquete até o de entrada na sala de transferência;

- o tempo de corredor ( $\mathrm{t}$ corredor), correspondente ao intervalo de tempo decorrido entre horário de entrada no corredor e horário de entrada na entrada na sala de transferência.

Para análise, o horário de entrada na sala de transferência (hora-dia) foi agrupado em três classes (às 13h, 15h e 17h).

$\mathrm{Na}$ sala de transferência, registraram-se: o número da receptora, a hora de chegada, a hora de saída (hh:mm), a duração do processo de inovulação (DURAÇÃO), a temperatura da sala $\left({ }^{\circ} \mathrm{C}\right)$ e a umidade relativa do ar (\%) medidas por meio de um termômetro digital logo após a entrada do animal. Foram mensuradas as seguintes variáveis clínicas: frequência respiratória/minuto (FR), observada através das oscila- ções do flanco do animal contada em quinze segundos e multiplicado por quatro; temperatura retal (T Retal), medida por um termômetro digital até que a temperatura se estabilizasse; temperatura da pele (T Pele), obtida com termômetro digital de superfície, posicionado na região das costelas, cerca de $20 \mathrm{~cm}$ atrás da paleta, 20 $\mathrm{cm}$ abaixo da linha do dorso, distante $3 \mathrm{~cm}$ do animal. Calculou-se, ainda, a diferença entre temperatura da pele e retal (DifT). Também foi registrada a pelagem do animal (branca, osco-claro, osco-escuro, brasina, vermelha, malhada de vermelho e branco e preta).

Quanto ao embrião recebido, foram anotados: o número do touro que inseminou as doadoras de embriões (TOURO), a partida do sêmen (PARTIDA), o número da doadora do embrião (DOADORA), o estágio de maturação do embrião (mórula compacta (MC), mórula (MO), blastocisto inicial (BI), blastocisto (BL) e blastocisto expandido (BX)), bem como a classificação da qualidade do embrião (excelente, bom, regular e pobre). Definiram-se o estágio de maturação do embrião e a classificação do embrião de acordo com a International Embryo Transfer Society (IETS, 1999), tendo sido transferidos apenas embriões grau 1 a 3 .

No tronco de contenção foram registrados os comportamentos em relação a mugidos: escore $0=$ sem ocorrência, escore 1 = ocorrência; respiração: escore 1 = não audível, escore 2 = audível e profunda, escore 3 = bufando/ roncando (respiração forçada com emissão de sons); postura: escore $1=$ em pé, escore 2 = ajoelhada, escore 3 = deitada; tensão: escore $1=$ relaxada (não apresenta movimentos abruptos de cauda e/ou cabeça), escore 2 = tensa (movimentos abruptos de cauda e/ou cabeça), escore 3 = muito tensa (apresenta tremor muscular). Com base nesses dados atribuiu-se um escore quanto à reatividade do animal (Quadro 1), adaptado de FORDYCE et al. (1982).

QUADRO 1. Critérios para definição do escore de reatividade do animal de acordo com os escores de tensão, respiração e mugido (adaptado de FORDYCE et al., 1982).

\begin{tabular}{|l|c|c|c|}
\hline \multirow{2}{*}{\multicolumn{1}{c|}{ Reatividade }} & \multicolumn{3}{c|}{ Escore } \\
\cline { 2 - 4 } & Tensão & Respiração & Mugido \\
\hline Sem reatividade aparente ou calmo & 1 & 1 ou 2 & 0 ou 1 \\
\hline Baixa reatividade ou inquieto & 1 (se respiração $\geq 2)$ & 1,2 ou 3 (se mugido=0) & 0 ou 1 \\
\hline Média reatividade ou perturbado & 1 (se mugido $\neq 0$ ) ou 2 & 1,2 ou 3 (se mugido=0) & 0 ou 1 \\
\hline Reativo ou muito perturbado & 2 (se mugido $\neq 0$ ) ou 3 & 1,2 ou 3 (se mugido=0) & 0 ou 1 \\
\hline Muito reativo ou perigoso & $3($ se mugido $\neq 0)$ & ---- & ---- \\
\hline
\end{tabular}


Realizou-se a transferência de embriões pelo método cirúrgico, no flanco, com sedação prévia e anestesia local. O diagnóstico de gestação e de sexagem fetal foi realizado pelo médico veterinário da fazenda, ao final de sessenta dias, via palpação transretal e com o auxílio de um aparelho de ultrassom.

Além disso, foram registradas nos dias da inovulação as seguintes variáveis meteorológicas: a temperatura de bulbo seco (TBS) e de bulbo úmido (TBU) do local, a cada hora, com o auxílio de um psicrômetro, e a temperatura de globo negro (tg), respectivamente. Com base nesses dados, calcularam-se o índice de temperatura e de umidade (ITU) nos períodos da manhã (ITUm), tarde (ITUt) e do dia (ITUdia). O ITUm foi calculado das $08 \mathrm{~h}$ às $11 \mathrm{~h}$, o ITUt das $13 \mathrm{~h}$ às $17 \mathrm{~h}$ e o ITUdia das $08 \mathrm{~h}$ às $17 \mathrm{~h}$, calculados pela seguinte expressão (SILVA, 2000): ITU $=$ ta $+0,36$ tpo $+41,5$ (expresso em $\mathrm{kPa}$ - quilo pascal), em que: ta $=$ temperatura do ar, ${ }^{\circ} \mathrm{C}$; tpo $=$ temperatura do ponto de orvalho, ${ }^{\circ} \mathrm{C} ; 41,5=$ constante,

Também foi calculado o índice de temperatura de globo e umidade (ITGU) obtido pela seguinte expressão (SILVA, 2000): ITGU $=\operatorname{tg}+0,36$ tpo + 41,5 (expresso em $\mathrm{kPa})$, em que: $\mathrm{tg}=$ temperatura do termômetro de globo, ${ }^{\circ} \mathrm{C}$; tpo $=$ temperatura do ponto de orvalho, ${ }^{\circ} \mathrm{C} ; 41,5=$ constante.

Com base nesse índice, procedeu-se ao cálculo da média do ITGU da manhã (ITGUm), da tarde (ITGUt), do dia inteiro (ITGUdia) e ITGU do horário da inovulação (ITGUin). O ITGUm foi calculado das $08 \mathrm{~h}$ às $11 \mathrm{~h}$, o ITGUt das $13 \mathrm{~h}$ às $17 \mathrm{~h}$ e o ITGU dia das $08 \mathrm{~h}$ às $17 \mathrm{~h}$. Esse índice considera a temperatura de globo negro, que expressa não só a temperatura radiante, mas também a ação conjunta de outros elementos climáticos, como umidade do ar e a velocidade do vento (SILVA, 2000). Outras variáveis como temperatura máxima (Tmax), temperatura mínima (Tmin), precipitação pluviométrica $(\mathrm{PPt})$, velocidade do vento (VV) e umidade relativa do ar (UR) foram cedidas pela Embrapa Gado de Corte - Campo Grande-MS.

Foram coletadas em tubos heparinizados amostras de sangue das receptoras do lote 2, totalizando 36 receptoras, nos dias D0, D9 e D16 para determinação das concentrações de cortisol, e para progesterona somente no D16, imediatamente após a contenção do animal no tronco pela punção da veia caudal mediana. Após centrifugação a 1500 rpm/15 minutos, estocou-se o plasma obtido a $-20^{\circ} \mathrm{C}$, para posterior determinação das concentrações plasmáticas de progesterona e cortisol.

Efetuaram-se as análises de cortisol e progesterona em duplicata, utilizando-se kits de dosagens imunoenzimáticas (Diagnostic System Laboratories, Inc.) no Laboratório de Fisiologia Animal da Faculdade de Zootecnia e Engenharia de Alimentos / USP, Campus de Pirassununga - SP. O coeficiente de correlação intraensaio foi de $3,9 \%$ e o interensaio de $2,2 \%$.

A taxa de gestação obtida foi comparada de acordo com as variáveis: lote, touro, maturação do embrião, classificação do embrião, pelagem, reatividade, variáveis discriminadas quanto à reatividade $\mathrm{e}$ processamento do embrião por teste de dispersão de frequência $\left(\mathrm{X}^{2}\right)$ utilizando-se o pacote estatístico SAS (1995). Compararam-se as variações fisiológicas, clínicas e duração de manejo pelo teste $t$ de Student, entre dois grupos, um composto pelas fêmeas gestantes e outro pelas não gestantes, considerando-se o nível de significância de 5\%. Foi realizada análise de Correlação de Pearson entre as variáveis ambientais e os índices de conforto térmico e manejo dentro do grupo de vacas gestantes $(n=62)$ e de vacas não gestantes $(n=40)$.

Para verificar possíveis variações ambientais entre os dias de coleta, submeteram-se os dados à análise de variância, pelo procedimento GLM do pacote estatístico SAS (2005), considerando efeitos fixos de hora do dia (hora/dia), lote, diagnóstico de gestação e a interação hora/dia*lote sobre a variável ITGU do horário de inovulação (ITGUin), tempo de curral, tempo de espera. Procedeu-se à comparação entre médias pelo Teste de Duncan. Foi realizada análise de regressão, logística que se adequa à análise da relação entre respostas discretas com variáveis exploratórias (STOKES et al., 2000), utilizando pacote estatístico SAS (2005). A regressão logística é frequentemente utilizada na análise da relação entre respostas discretas com variáveis exploratórias (STOKES et al., 2000). Com a metodologia, objetivou-se modelar a probabilidade ( $\mathrm{p}$ ) de as matrizes serem classificadas como prenhes (1), em função das variáveis: lote, touro, classificação do embrião, tempo de manejo, tempo de curral, tempo de corredor, hora/dia, ITGU13, ITGU15, ITGU17, ITUT e ITUMD, e as variáveis clínicas: FR, T Retal, T Pele, DifT. 


\section{RESULTADOS}

Do total de 94 fêmeas, $62,8 \%$ foram diagnosticadas como gestantes e $37,2 \%$ como não gestantes, ao final de sessenta dias.

As médias das variáveis climáticas TBS, TBU, TG, VV, PPt, UR, Tmin, Tmax e Tméd, nos dias das inovulações, encontram-se sumarizadas na Tabela 1.
Verificou-se correlação negativa entre temperatura média do ar, vv, ITGUt e ITGUdia e a Dift, sendo esta significativa tanto para fêmeas gestantes como para as não gestantes (Tabela 2).

Os valores médios do ITGU e do ITU referentes ao dia da inovulação das receptoras são apresentados na Tabela 3.

TABELA 1. Variáveis meteorológicas referentes aos dias de inovulação das receptoras de embrião

\begin{tabular}{|c|c|c|c|c|c|c|c|c|c|}
\hline Data da inovulação & $\begin{array}{l}\text { TBS } \\
\left({ }^{\circ} \mathrm{C}\right)\end{array}$ & $\begin{array}{l}\text { TBU } \\
\left({ }^{\circ} \mathrm{C}\right)\end{array}$ & $\begin{array}{c}\mathrm{TG} \\
\left({ }^{\circ} \mathrm{C}\right)\end{array}$ & $\begin{array}{c}\mathrm{VV} \\
(\mathrm{m} / \mathrm{s})\end{array}$ & $\begin{array}{c}\mathrm{PPt} \\
(\mathrm{mm})\end{array}$ & $\begin{array}{l}\text { UR } \\
(\%)\end{array}$ & $\begin{array}{l}\text { Tmin } \\
\left({ }^{\circ} \mathrm{C}\right)\end{array}$ & $\begin{array}{c}\mathrm{T} \max \\
\left({ }^{\circ} \mathrm{C}\right)\end{array}$ & $\begin{array}{c}\text { Tméd } \\
\left({ }^{\circ} \mathrm{C}\right)\end{array}$ \\
\hline 26/02/04 (lote1) & 32,3 & 25,6 & 49,0 & $3,1^{\mathrm{a}}$ & $0,0^{\mathrm{b}}$ & $59^{c}$ & 21,5 & 33,9 & $26,5^{b}$ \\
\hline 02/03/04 (lote2) & 29,7 & 25,9 & 47,8 & $3,0^{\mathrm{b}}$ & $0,0^{\mathrm{b}}$ & $63^{b}$ & 23,7 & 35,2 & $28,4^{\mathrm{a}}$ \\
\hline 16/03/04 (lote3) & 31,3 & 26,0 & 46,2 & $1,2^{\mathrm{c}}$ & $11,7^{\mathrm{a}}$ & $74^{a}$ & 21,7 & 32,7 & $26,2^{b}$ \\
\hline
\end{tabular}

TBS: Temperatura do bulbo seco; TBU: temperatura do bulbo úmido; TG: temperatura do globo negro; (Variáveis coletadas na Fazenda); VV: velocidade do vento; PPt: precipitação pluviométrica; UR: umidade relativa do ar; Tmin: temperatura mínima; Tmax: temperatura máxima; Tméd: temperatura média; (Médias diárias obtidas em Relatórios da Embrapa Gado de Corte-CNPGC, no ano de 2004). Letras diversas na mesma coluna indicam diferença significativa em nível de $5 \%$ pelo teste de Duncan.

TABELA 2. Correlação de Pearson entre as variáveis climáticas, índice de temperatura de globo e umidade e a diferença entre a temperatura de pele e retal (DifT) de acordo com o estado gestacional das receptoras

\begin{tabular}{lll}
\hline & Gestante $($ valor de $\mathrm{p})$ & Não gestante $($ valor de $\mathrm{p})$ \\
\hline Temperatura do ar vs Dift & $-0,54(\mathrm{p}<0,0001)$ & $-0,63(\mathrm{p}<0,0001)$ \\
ITGUt $v$ s Dift & $-0,40(\mathrm{p}<0,005)$ & $-0,54(\mathrm{p}<0,001)$ \\
ITGUdia $v s$ Dift & $-0,40(\mathrm{p}<0,005)$ & $-0,59(\mathrm{p}<0,001)$ \\
VV $v$ DifT & $-0,41(\mathrm{p}<0,005)$ & $-0,58(\mathrm{p}<0,001)$ \\
\hline
\end{tabular}

DifT: diferença entre temperatura da pele e retal; ITGUt: índice de temperatura de globo e umidade da tarde; ITGUdia: índice de temperatura de globo e umidade do dia; VV: velocidade do vento.

TABELA 3. Valores médios dos índices de temperatura de globo e umidade (ITGU) e índice de temperatura e umidade (ITU) referentes ao dia da inovulação das receptoras

\begin{tabular}{cccccccc}
\hline Lotes & $\begin{array}{c}\text { Data da inovu- } \\
\text { lação }\end{array}$ & ITGU manhã & ITGU tarde & ITGU dia & ITU manhã & ITU tarde & ITU dia \\
\hline Lote 1 & $26 / 02 / 04$ & $94,47^{\mathrm{c}}$ & $97,61^{\mathrm{a}}$ & $96,21^{\mathrm{a}}$ & $77,65^{\mathrm{b}}$ & $81,61^{\mathrm{a}}$ & $79,18^{\mathrm{a}}$ \\
Lote 2 & $02 / 03 / 04$ & $95,63^{\mathrm{b}}$ & $94,99^{\mathrm{b}}$ & $96,21^{\mathrm{a}}$ & $78,47^{\mathrm{a}}$ & $78,78^{\mathrm{c}}$ & $76,49^{\mathrm{a}}$ \\
Lote 3 & $16 / 03 / 04$ & $97,74^{\mathrm{a}}$ & $89,80^{\mathrm{c}}$ & $93,77^{\mathrm{b}}$ & $77,52^{\mathrm{b}}$ & $80,00^{\mathrm{b}}$ & $78,63^{\mathrm{a}}$ \\
\hline
\end{tabular}

Manhã: média dos índices de conforto térmico das $7 \mathrm{~h}$ às $11 \mathrm{~h}$; tarde: média dos ITUs das $12 \mathrm{~h}$ às $17 \mathrm{~h}$; dia: média do dia ( $7 \mathrm{~h}$ às $17 \mathrm{~h})$. Letras diversas na mesma coluna indicam diferença significativa em nível de $5 \%$ pelo teste de Duncan.

O modelo de análise adotado foi significativo para as seguintes variáveis: ITGUin (Anova: $\mathrm{P}<0,0001$; $\left.\mathrm{R}^{2}=0,95 ; \mathrm{CV}=1,84\right)$, considerando os efeitos de lote
( $\mathrm{P}<0,001)$, hora/dia $(\mathrm{P}<0,001)$ e interação lote*hora/ dia $(\mathrm{P}<0,001)$; tempo de espera (Anova: $\mathrm{P}<0,0001$; $\left.\mathrm{R}^{2}=0,88 ; \mathrm{CV}=18,76\right)$, comparando-se com lote 
$(\mathrm{P}=0,004)$, hora/dia $(\mathrm{P}<0,001)$ e interação lote*hora/ dia $(\mathrm{P}=0,002)$ e tempo de manejo (Anova: $\mathrm{P}<0,0001$; $\left.\mathrm{R}^{2}=0,64 ; \mathrm{CV}=10,47\right)$, considerando os efeitos de lote $(\mathrm{P}<0,004)$, hora/dia $(\mathrm{P}<0,001)$ e interação lote*hora/ dia $(\mathrm{P}=0,002)$; não sendo significativo para diagnóstico de gestação.

A distribuição do ITGU dos três lotes de acordo com o horário da inovulação é ilustrada na Figura 1.

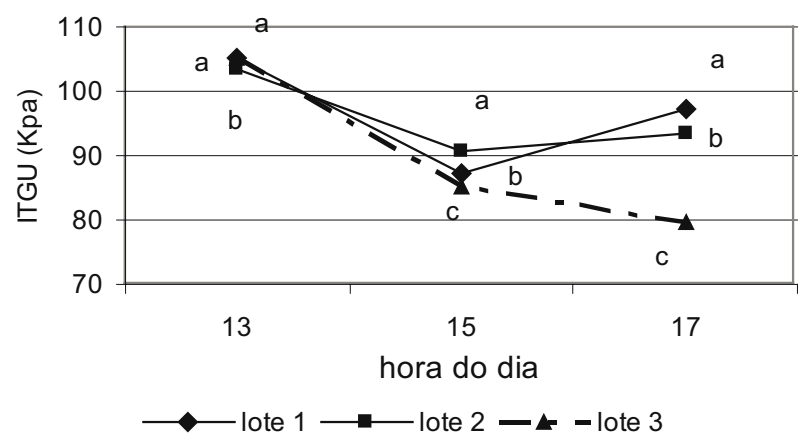

FIGURA 1. Índice de temperatura de globo e umidade médio registrado em diferentes horários de realização da inovulação (ITGUin) em fêmeas bovinas receptoras de acordo com o lote trabalhado: lote $1(26 / 02 / 04)$, lote $2(02 / 03 / 04)$ e lote $3(16 / 03 / 04)$. Letras diversas no mesmo horário indicam diferença significativa em nível de $5 \%$ de significância pelo teste de tukey.

O tempo de manejo médio das fêmeas, em minutos, foi de 417,22 $\pm 70,09$ e 394,11 $\pm 46,47$ e o tempo de corredor foi de 65,86 $\pm 48,06$ e 62,91 $\pm 43,11$ para as fêmeas gestantes e não gestantes, respectivamente (Tabela 4), não havendo diferença significativa $(\mathrm{P}>0,05)$ pelo teste $\mathrm{t}$ de student. A análise de regressão logística demonstrou que a probabilidade de prenhez das receptoras de embriões poderia ser explicada por uma equação que considerasse o efeito fixo do horário de entrada para a inovulação. A associação da probabilidade estimada e resposta observada mostrou uma percentagem de concordância de $68,2 \%$ e percentagem de discordância $31,1 \%$, o que permite verificar que o modelo (equação) concordou com os dados observados, ou seja, o modelo estimado possui uma boa aderência com os dados observados. Na situação trabalhada, houve uma tendência $(\mathrm{P}=0,08)$ de variação da probabilidade prenhez de cerca de $25 \%$ entre o início e o final da tarde, como ilustrado na Figura 2.
TABELA 4. Caracterização do manejo (tempo de espera no curral e tempo de espera no corredor de acesso à sala de transferência média e desvio-padrão) de fêmeas bovinas receptoras de embriões de acordo com o diagnóstico de gestação.

\begin{tabular}{ccc}
\hline & Gestantes & Não gestantes \\
\hline $\begin{array}{c}\text { t curral (minutos) } \\
(\text { min - max })\end{array}$ & $\begin{array}{c}417,22 \pm 70,09^{\mathrm{a}} \\
(398,95-435,49)\end{array}$ & $\begin{array}{c}394,11 \pm 46,47^{\text {a }} \\
(378,15-410,08)\end{array}$ \\
& & \\
$\mathrm{t}$ corredor (minutos) & $65,86 \pm 48,06^{\mathrm{a}}$ & $62,91 \pm 43,11^{\mathrm{a}}$ \\
$(\min -\max )$ & $(53,34-78,39)$ & $(48,11-77,72)$ \\
\hline
\end{tabular}

Letras diversas na mesma linha indicam diferença significativa pelo teste $\mathrm{t}$ de student em nível de 5\% de significância.

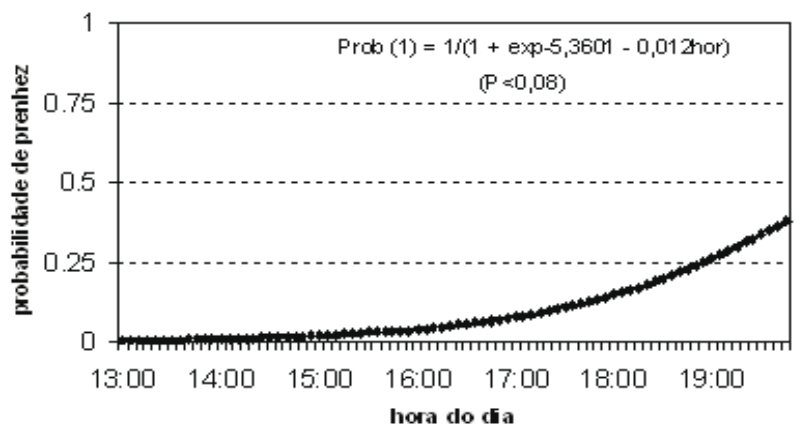

FIGURA 2. Probabilidade de prenhez de vacas receptoras de embriões em relação ao horário de entrada na sala de transferência em uma propriedade no Brasil Central. $(\operatorname{Prob}(1)=1 /(1+\mathrm{e}-5,3601-$ 0,012hor), $\mathrm{P}<0,08$ pelo teste $\mathrm{t}$ ).

Não houve diferença significativa $(\mathrm{P}>0,05)$, pelo teste do $\mathrm{X},{ }^{2}$ entre as fêmeas gestantes e não gestantes com relação ao estágio de maturação do embrião, classificação da qualidade do embrião, pelagem, reatividade, postura, mugido, respiração, tensão e entre embriões congelados e a fresco.

Com relação aos sinais clínicos, as médias de FR, Tretal e a diferença entre temperatura da pele e retal não diferiram $(\mathrm{P}>0,05)$ pelo teste $\mathrm{t}$ de Student entre as fêmeas diagnosticadas como gestantes e não gestantes. Entretanto, houve diferença significativa entre temperatura da pele de gestantes e não gestantes $(\mathrm{P}<0,05)$ (Tabela 5).

As concentrações médias de cortisol para os dias D0, D9 e D16 foram 19,13 $\pm 7,48 ; 16,32 \pm 7,89$ e 14,56 $\pm 8,02 \mathrm{ng} / \mathrm{mL}$, respectivamente (Figura 3). 
Observou-se diferença significativa apenas entre os dias DO e D16 $(\mathrm{P}<0,05)$.

Não foi observada diferença significativa nos níveis de cortisol no decorrer do protocolo quando se compararam as médias das fêmeas gestantes separadamente das não gestantes. As concentrações médias de cortisol para os dias D0, D9 e D16 das fêmeas gestantes foram $19,91 \pm 7,44 ; 16,25 \pm 7,96$ e 14,66 $\pm 7,83 \mathrm{ng} /$ $\mathrm{mL}$, respectivamente, e das fêmeas não gestantes fo$\operatorname{ram} 17,89 \pm 7,77 ; 16,45 \pm 8,12$ e $14,38 \pm 8,73 \mathrm{ng} / \mathrm{mL}$, respectivamente (teste $t$ de Student, $\mathrm{P}>0,05$ ).

TABELA 5. Aspectos clínicos de receptoras bovinas no momento da inovulação de embriões Nelore de acordo com resultado de prenhez

\begin{tabular}{lcc}
\hline Dados clínicos & Gestantes & Não gestantes \\
\hline Frequência respiratória (mov/ & $48,44 \pm 9,50^{\text {a }}$ & $49,14 \pm 9,48^{\text {a }}$ \\
min) & $39,75 \pm 0,47^{\text {a }}$ & $39,79 \pm 0,51^{\text {a }}$ \\
Temperatura retal $\left({ }^{\circ} \mathrm{C}\right)$ & $37,31 \pm 0,63^{\mathrm{b}}$ & $37,78 \pm 1,05^{\text {a }}$ \\
Temperatura da pele $\left({ }^{\circ} \mathrm{C}\right)$ & $2,53 \pm 0,75^{\text {a }}$ & $2,54 \pm 0,96^{\text {a }}$ \\
\hline
\end{tabular}

DifT: diferença entre temperatura de pele e retal $\left({ }^{\circ} \mathrm{C}\right)$. Letras diferentes na mesma linha indicam diferença significativa pelo teste $\mathrm{t}$ de Student em nível de 5\% de significância.

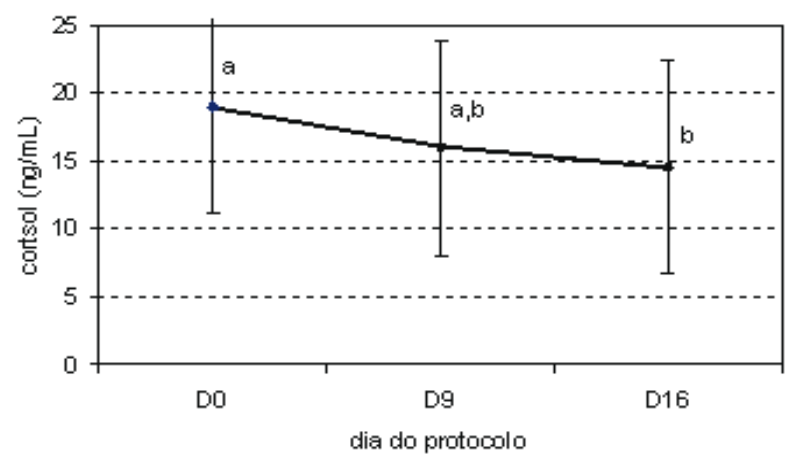

FIGURA 3. Curva da concentração média de cortisol plasmático (ng/mL) das receptoras de embriões do lote 2 nos dias D0, D9 e D16. Letras diversas indicam diferença significativa pelo teste $t$ de Student $(\mathrm{P}<0,05)$.

As concentrações médias de progesterona no dia D16 das fêmeas gestantes e não gestantes foram $35,78 \pm 19,07$ e $28,59 \pm 17,55 \mathrm{ng} / \mathrm{mL}(\mathrm{P}<0,05)$, respectivamente, e de cortisol foram $14,66 \pm 7,83 \mathrm{e}$ $14,38 \pm 8,73 \mathrm{ng} / \mathrm{mL}(\mathrm{P}>0,05)$ (Figura 4). Não houve correlação $(\mathrm{P}>0,05)$ entre cortisol e progesterona no dia da inovulação para gestantes $(0,39)$ e não gestantes $(0,27)$, respectivamente.

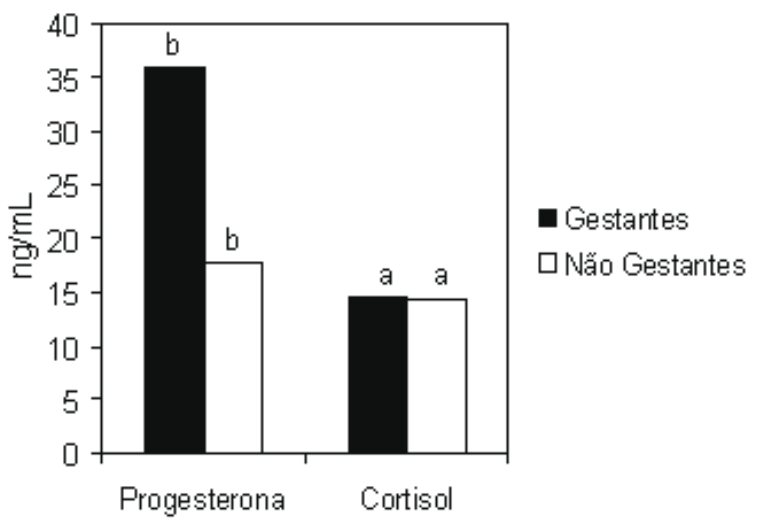

FIGURA 4. Concentração média de progesterona e cortisol plasmático $(\mathrm{ng} / \mathrm{mL})$ do lote 2 no dia da inovulação das receptoras de embriões gestantes e não gestantes. Letras diversas indicam diferença significativa em nível de 5\% pelo teste $t$ de Student.

\section{DISCUSSÃO}

Encontrou-se uma taxa de gestação aos sessenta dias pós-inovulação de $62,77 \%$, valor acima dos relatados aos 45 dias pós-inovulação por VASCONCELOS et al. (2006), de 45,80\%, e DROST et al. (1999), de $35,40 \%$, e compatíveis aos encontrados por REICHENBACH et al. (2002), que observaram taxas médias de gestação em torno de $60 \%$ em transferências de embriões a fresco de grau 1 a 3, classificados de acordo com a IETS (1999).

A capacidade do animal de resistir ao estresse por calor, em função de altas temperatura e umidade relativa do ar, tem sido avaliada fisiologicamente por alterações na temperatura retal e na frequência respiratória, explicando a tentativa de o animal perder o calor absorvido (AZEVEDO et al., 2005; MAIA et al., 2005). A temperatura retal não diferiu estatisticamente entre gestantes e não gestantes, como já havia sido observado por KATAYAMA et al. (2004), na mesma propriedade usada por este estudo no mês de novembro, contrastando com VASCONCELOS et al. (2006), que observaram, em vacas Holandesas receptoras de embriões, que o aumento da temperatura retal no sétimo dia após 
o estro diminuiu a taxa de prenhez no dia $25(\mathrm{P}<0,05)$ e aumentou a perda embrionária $(\mathrm{P}<0,10)$. Entretanto, a temperatura retal média das receptoras estava acima da normalidade, de acordo com a citada por DUKES (1996), em que o valor máximo para bovinos de corte é de $39,1^{\circ} \mathrm{C}$. Experimentos in vivo demonstraram que o estresse por calor no primeiro dia de prenhez reduziu a sobrevivência do embrião e a taxa de prenhez. Entretanto, a partir do terceiro dia o estresse por calor não afetou essa taxa (EALY et al., 1993), indicando que embriões bovinos se tornam mais resistentes aos efeitos prejudiciais do estresse por calor materno com o avanço do seu estágio de desenvolvimento.

A maior temperatura de pele das fêmeas não gestantes observada neste estudo sugere uma influência negativa sobre a taxa de gestação, demonstrando que a dificuldade em perder calor, com consequente aumento de temperatura interna, pode ter prejudicado o desenvolvimento embrionário, como já observado por MALAYER \& HANSEN (1990). A temperatura de pele pode ser considerada um bom indicador da atividade de sudorese em bovinos (SHARF et al., 2008). A termólise evaporativa é o mais efetivo meio da dissipação de calor corporal, sendo que a evaporação cutânea representa cerca de $85 \%$ da perda total do calor latente (MAIA et al., 2005). A queda da temperatura de pele relacionada ao aumento do estresse ocorre quando há estimulação simpática, o que gera vasoconstrição periférica e o aumento da pressão arterial, levando a um aumento da temperatura interna e à diminuição da temperatura periférica do corpo (RANDALL et al., 2002). O fato de as vacas prenhas terem apresentado temperatura de pele menor que as vacas que não ficaram gestantes sugere que estas estariam acionando de forma mais eficiente os mecanismos de termólise evaporativa cutânea.

A não observância de diferença significativa na difT contrastou com os dados encontrados por KATAYAMA et al. (2004). Os autores trabalharam na mesma propriedade utilizada neste estudo no mês de novembro, encontrando diferença significativa entre fêmeas gestantes e não gestantes $\left(4,64^{\circ} \mathrm{C}\right.$ e $3,62^{\circ} \mathrm{C}$, respectivamente, $\mathrm{P}<0,05)$ e mostrando uma maior eficiência para perda de calor das fêmeas gestantes. Essa diferença pode ter ocorrido pelas variações ambientais externas nos períodos avaliados nos dois trabalhos quanto a fatores que favorecem a termóli- se como umidade ou velocidade e direção do vento (SILVA, 2000).

Quanto à frequência respiratória, não houve diferença significativa entre fêmeas gestantes e não gestantes. Entretanto ambas estavam superiores à normalidade, que é de 29 movimentos por minuto para vacas leiteiras em estação (DUKES, 1996), indicando que essas fêmeas ativaram mecanismos fisiológicos para perda de calor para atingir a homeostase.

Avaliaram-se o tempo de manejo das fêmeas e o tempo de corredor de acesso à sala de transferência como um fator estressante para essas fêmeas. Não houve diferença significativa entre as gestantes e não gestantes tanto para tempo de manejo quanto para tempo de corredor, diferindo dos resultados encontrados por KATAYAMA et al. (2004), que encontraram diferença significativa $(\mathrm{P}<0,05)$ no tempo de espera no curral $(3 \mathrm{~h} 28 \mathrm{~m}$ e $2 \mathrm{~h} 50 \mathrm{~m}$ para fêmeas não gestantes e gestantes, respectivamente). Isto aconteceu provavelmente porque os animais do experimento realizado por KATAYAMA et al. (2004), após o exame ginecológico, aguardavam em um curral sem sombra e sem água até o momento da inovulação. Já neste experimento, as fêmeas aguardavam em um piquete com condições opostas às dos autores citados. Essa diferença no manejo muito provavelmente minimizou o efeito do estresse, o que pode ser reforçado pelo fato de as fêmeas, trabalhadas no final da tarde, em horários com ITGU significativamente mais baixo, terem apresentado maior probabilidade de prenhez (Figura 3 ).

Segundo CHEBEL et al. (2004), vacas expostas ao estresse por calor ao menos um dia de temperatura máxima $>29^{\circ} \mathrm{C}$ antes da inseminação artificial tiveram taxa de gestação menor $(\mathrm{p}<0,001)$ que vacas não expostas a esse fator. Entretanto, nos achados deste experimento, apesar de a temperatura e a umidade relativa do ar estarem elevadas, não houve diferença significativa quanto à taxa de prenhez, uma vez que todos os lotes foram submetidos às condições de conforto térmico acima dos valores críticos. Os valores de ITU encontrados neste trabalho foram acima de 77 , indicando uma situação de desconforto térmico para os animais, independente da condição de prenhez positiva ou não. De acordo com HAHN (1985), na escala de aceitabilidade para ITU, feita para condições de clima temperado, um valor de ITU menor que 70 é considerado uma condição de conforto térmico; va- 
lores entre 71 e 78 são considerados críticos; entre 79 e 83 indicam perigo; acima de 83 já constituem uma situação de emergência.

Quanto ao ITGU, não existem critérios definidos para avaliar o desconforto térmico dos animais (SILVA et al., 2006), mas neste estudo os valores estavam acima de 93 e atingiram picos entre às 10 e 14 horas, quando o desconforto foi mais intenso.

O estudo mostrou um declínio nas concentrações de cortisol nos dias D0, D9 e D16 e diferença significativa entre o primeiro dia de manejo e o dia da inovulação (D0 vs D16), sugerindo que houve uma habituação dos animais ao manejo da fazenda, ou seja, diminuição da resposta de medo ao estímulo estressante, o que refletiria na queda de liberação do cortisol observada (RODRIGUES et al., 2009). Vacas Friesian, em repouso, possuem nível basal de cortisol plasmático de $2 \mathrm{ng} / \mathrm{mL}$ (ALAM \& DOBSON, 1986). Fêmeas holandesas, durante o manejo de ordenha, apresentaram cortisol em torno de $13 \mathrm{ng} / \mathrm{mL}$, assim como as que são presas no tronco de contenção durante um manejo no curral (LAY et al., 1992). Em zebuínos, ANDRADE et al. (2001) encontraram cortisol plasmático de 3,92 $\pm 1,20 \mathrm{ng} / \mathrm{mL}$, podendo decrescer a níveis médios de $0,99 \pm 0,37 \mathrm{ng} / \mathrm{mL}$ em vacas habituadas à rotina de manejo. As concentrações plasmáticas nas receptoras de embriões estavam acima desses parâmetros fisiológicos nos dois grupos, gestantes e não gestantes. Assim, provavelmente, todas as receptoras estavam sob condições de estresse elevado, o que pode ser devido aos manejos frequentes e também às condições de desconforto térmico, caracterizadas pelos índices de conforto a que foram submetidas sempre em níveis críticos.

Níveis elevados de cortisol inibem a secreção de GnRH pelo hipotálamo e de LH pela hipófise, essencial na fase folicular e pré-ovulatória (MacFARLANE et al., 2000; BREEN et al., 2005). O cortisol pode agir negativamente sobre a função lútea, e consequentemente nas concentrações de progesterona, como encontrado por LEYVA-OSCARIZ et al. (1996), que observaram que cortisol associado com a estação seca pode diminuir a progesterona secretada pelo corpo lúteo em vacas da raça Carora em clima tropical. EDWARDS et al. (1987) observaram, em novilhas doadoras de embriões transportadas por quinze a sessenta minutos a cada doze horas por quatro dias, que a concentração plasmática de cortisol foi maior nas fêmeas "estressadas" em comparação às fêmeas-controle $(34,8 \pm 2,2$ vs $28,4 \pm 2,5 \mathrm{ng} / \mathrm{mL}, \mathrm{P}<0,1)$. Entretanto, neste estudo, não houve correlação entre cortisol e progesterona no dia da inovulação para gestantes e não gestantes, como verificado por WILLARD et al. (2005).

A progesterona tem a função reguladora do ciclo estral e representa o hormônio essencial da gestação. Em todas as espécies, a fonte de progesterona está garantida no início da gestação pelo corpo lúteo, que na vaca conserva sua função durante toda a gestação. A alta concentração de progesterona está relacionada com baixos níveis de estrógeno e com a inibição da secreção de hormônios luteolíticos como a prostaglandina (DUKES, 1996; GALIMBERTI et al., 2001).

DROST et al. (1999) consideravam a prenhez aos 22 dias pós-inovulação quando níveis de progesterona estivessem acima de $1 \mathrm{ng} / \mathrm{mL}$. Já PINHO et al. (1988) consideraram níveis acima de 2,0ng/mL aos 17 dias pós-inseminação artificial. GREGORY et al. (1986) citaram que receptoras que apresentaram níveis de progesterona acima de $4 \mathrm{ng} / \mathrm{mL}$ tiveram maior probabilidade de se tornarem prenhes, considerando embriões de mesma qualidade. Neste trabalho, as concentrações de progesterona foram significativamente diferentes para as receptoras gestantes e não gestantes, assim como relatado por PINHO et al. (1988), e em ambas, gestantes e não gestantes, as concentrações estavam superiores a $4 \mathrm{ng} / \mathrm{mL}$. O aumento da progesterona se deve a um estímulo da função luteínica dos corpos lúteos resultante da ovulação de folículos estimulados pelo eCG (BARUSELLI et al., 2004) e também pela secreção da adrenal, como observado por WILLARD et al. (2005). Esses autores verificaram que o aumento agudo de ACTH elevou a progesterona de origem adrenal no meio da gestação $(139,0 \pm 5$ dias $)$ de novilhas Brahman. NASSER et al. (2004) verificaram que houve aumento da progesterona plasmática decorrente do tratamento com eCG no quinto dia do ciclo estral, aumentando a taxa de prenhez de receptoras de embriões.

Como todas as receptoras estavam sujeitas às mesmas condições térmicas, caracterizadas na fase experimental como críticas para bovinos, as variações individuais na tentativa de alcançar a homeostase provavelmente influenciaram a fertilidade das receptoras bovinas. Dentre as variáveis fisiológicas avaliadas 
neste experimento, a temperatura da pele e a concentração plasmática de progesterona variaram em função das respostas individuais de ajuste térmico. A temperatura de pele pode ser indicativa do desconforto térmico que influenciou na taxa de gestação das receptoras de embriões. E a variação dos níveis plasmáticos de progesterona muito provavelmente seria um reflexo das variações individuais de resposta à condição de estresse (MacFARLANE et al., 2000; BREEN et al., 2005). A observação de aumento da probabilidade de prenhez nas fêmeas inovuladas no final da tarde reforça os indicativos de influência do estresse térmico sobre a fertilidade de vacas receptoras de embriões. Os resultados observados neste experimento sugerem que a adaptação ao calor é um fator importante a ser observado na seleção de receptoras, e a temperatura de pele é uma medida simples que pode ser utilizada um critério de seleção.

\section{CONCLUSÕES}

O estresse térmico por calor afetou a probabilidade de prenhez de vacas receptoras de embriões sob condições tropicais. A temperatura de pele e os níveis plasmáticos de progesterona no momento da inovulação foram as variáveis fisiológicas que afetaram a probabilidade de gestação das receptoras. A temperatura de pele é uma medida simples que pode ser adotada como critério de seleção de vacas receptoras que entrarão em programas de transferência de embriões. Dentre as medidas de manejo, a opção de realizar a inovulação nos horários mais frescos pode ser uma forma de favorecer a regulação térmica das receptoras.

\section{AGRADECIMENTOS}

À Fundação de Apoio ao Desenvolvimento do Ensino, Ciência e Tecnologia - MS, pelo apoio financeiro e concessão de bolsas de mestrado; ao Conselho Nacional de Desenvolvimento Científico e Tecnológico (CNPq), pela cessão de bolsas de iniciação científica, e à Fazenda Sete Estrelas Embriões, pelo fornecimento dos animais, estrutura e recursos financeiros.

\section{REFERÊNCIAS}

ALAM, M. G. S.; DOBSON, H. Effect of various veterinary procedures on plasma concentrations of cortisol, luteinizing hormone and prostaglandin E2 metabolite in the cow. Veterinary Record, v. 118, p. $7-10,1986$.

ANDRADE, O.; ORIHUELA, A.; SOLANO, J.; GALINA, C. $\mathrm{S}$. Some effects of repeated handling and the use of a mask on stress responses in zebu cattle during restraint. Applied Animal Behaviour Science, v. 71, p. 175-181, 2001.

AZEVEDO, M.; PIRES, M. F. A.; SATURNINO, H.; LANA, A. M. Q.; SAMPAIO, I. B. M.; MONTEIRO, J. B. N.; MORATO, L. E. Estimativa de níveis críticos superiores do índice de temperatura e umidade para vacas leiteiras 1/2,3/4 e 7/8 Holandês-Zebu e lactação. Revista Brasileira de Zootecnia, v. 34, n. 6, p. 2000-2008, 2005.

BARUSELLI, P. S.; REIS, E. L.; MARQUES, M. O.; NASSER, L. F.; BÓ, G. A. The use of hormonal treatments to improve reproductive performance of anestrous beef cattle in tropical climate. Animal Reproduction Science, v. 82-83, s/n, p. 479-486, 2004.

BREEN, K. M.; BILLINGS, H. J.; WAGENMAKER, E. R.; WESSINGER, E. W.; KARSCH, F. J. Endocrine basis for disruptive effect of cortisol on preovulatory events. Endocrinology, v. 146, n. 4, p. 2107-2115, 2005.

CHEBEL, R. C.; SANTOS, J. E. P.; REYNOLDS, P. P.; CERRI, R. L.; JUCHEM, S. O.; OVERTON, M. Factors affecting conception rate after artificial insemination and pregnancy loss in lactating dairy cows. Animal Reproduction Science, v. 84, p. 239-255, 2004.

DROST, M.; AMBROSE, J. D.; THATCHER, M-J.; CANTRELL, C. K.; WOLFSDORF, K. E.; HASLER, J. F.; THATCHER, W. W. Conception rates after artificial insemination or embryo transfer in lactating dairy cows during summer in Florida. Theriogenology, v. 52, p. 1161-1167, 1999.

DUKES, H. H. Fisiologia dos animais domésticos. 11. ed. Rio de Janeiro: Guanabara Koogan, 1996. 856 p.

EALY, A. D.; DROST, M.; HANSEN, P. J. Developmental changes in embryonic resistance to adverse effects of maternal heat stress in cows. Journal Dairy Science, v. 76, p. 2899-2905, 1993.

EDWARDS, L. M.; RAHE, C. H.; GRIFFIN, J. L; WOLFE, D. F.; MARPLE, D. N.; CUMMINS, K. A.; PITCHETT, J. F. Effect of transportation stress on ovarian function in superovulated Hereford heifers. Theriogenology, v. 28, n. 3, p. 291-299, 1987.

FORDYCE, G.; GODDARD, M. E.; SEIFERT, G. W. The measurement of temperament in cattle and the effect of experience and genotype. Australian Society Animal Production, v. 14, p. 329-32, 1982.

GALIMBERTI, A. M.; FONSECA, F. A.; ARAÚJO, M. C. C.; COSTA, E. P.; FREITAS, C; GUIMARÃES, J. D.; FERREIRA, A. 
M. Taxa de gestação e níveis plasmáticos de progesterona, em receptoras de embrião bovino, tratadas com buserelina após a inovulação. Revista Brasileira de Zootecnia, v. 30, n. 2, p. 353-359, 2001.

GREGORY, R. M.; GAMBARINI, M. L.; BOLZONI, P. A. Concentração de progesterona sérica e índice de gestação em vacas receptoras de embriões. In: REUNIÃO ANUAL DA SOCIEDADE BRASILEIRA DE TRANSFERÊNCIA DE EMBRIÕES, 1., 1986. Jaboticabal-SP. Anais... Jaboticabal: SBTE, 1986. p. 98.

HAHN, G. L. Management and housing of farm animals in hot environments. In: YOUSEF, M. K. (Ed.). Stress physiology in livestock. Boca Raton: CRC Press, 1985. v. 2, p. 151-174.

IETS. Manual of the International Embryo Transfer Society. Savoy: IETS, 1999.

KATAYAMA, K. A.; MACEDO, G. G.; TZEIMAZIDES, S. P.; RUEDA, P. M.; REZENDE, C. R. L.; ZÚCCARI, C. E. S. N.; COSTA e SILVA, E. V. Manejo de receptoras bovinas em programas de transferência de embriões e taxas de gestação: resultados preliminares. In: REUNIÃO ANUAL DA SOCIEDADE BRASILEIRA DE ZOOTECNIA, 41., 2004, Campo Grande-MS. Anais... Campo Grande: Embrapa Gado de Corte, 2004. CD-ROM. Ambiência, Bioclimatologia e Etologia. AMB048. Disponível em <www.sbz. org.br/cds/SB22004.rar>

LANDAETA-HERNANDEZ, A. J.; YELICH, J.; LEMASTER, J. W. Environmental, genetic and social factors affecting the expression of estrus in beef cows. Theriogenology, v. 57, p. 1357-1370, 2002.

LAY, D. C.; FRIEND, T. H. JR.; BOWERS, C. L.; GRISSOM, K. K.; JENKINS, O. C. A comparative physiological and behavioral study of freeze and hot-iron branding using dairy cows. Journal Animal Science, v. 70, p. 1121-1125, 1992.

LEYVA-OSCARIZ, H.; QUERALES, G.; SAAVEDRA, J.; HERNÁNDEZ, A. Habeas luteum activity, fertility, and adrenal cortex response in lactating Carora cows during rainy and dry seasons in tropics of Venezuela. Domestic Animal Endocrinology, v. 13, n. 4, p. 297-306, 1996.

MACFARLANE, M. S.; BREEN, K. M.; SAKURAI, H.; ADAMS, B. M.; ADAMS, T. E. Effect of duration of infusion of stress-like concentrations of cortisol on follicular development and the preovulatory surge of LH in sheep. Animal Reproduction Science, v. 63 , p. $167-175,2000$.

MAIA, A. S. C.; SILVA, R. G.; LOUREIRO, C. M. B. Sensible and latent heat loss form the body surface of holstein cow in a tropical environment. International Journal of Biometeorology, v. 50, p. 17-22, 2005.

MALAYER, J. R.; HANSEN, P. J. Differences between Brahman and Holstein cows in heat-shock induced alterations of synthesis and secretion by oviducts and uterine endometrium. Journal Animal Science, v. 68, p. 266-280, 1990.

MANUAL OF THE INTERNATIONAL EMBRYO TRANSFER SOCIETY. Savoy: IETS; 1999.

MORAIS D. A. E. F.; MAIA, A. S. C.; SILVA, R. G.; VASCONCELOS, A. M.; LIMA, P. O.; GUILHERMINO, M. M. Variação anual de hormônios tireoideanos e características termorreguladoras de vacas leiteiras em ambiente quente. Revista Brasileira de Zootecnia, v. 37, n. 3, p. 538-545, 2008.

NASSER, L. F.; REIS, E. L.; OLIVEIRA, M. A.; BÓ, G. A.; BARUSELLI, P. S. Comparison of four synchronization protocols for fixed-time bovine embryo transfer in Bos indicus x Bos taurus. Theriogenology, v. 62, n. 9, p. 1577-1584, 2004.

NIEMANN, H.; SACHER, B.; ELSAESER, F. Pregnancy rates relative to recipient plasma progesterone levels on the day of no surgical transfer of frozen/thawed bovine embryos. Theriogenology, v. 23, p. 631-639, 1985.

ORIHUELA, A. Some factors affecting the behavioural manifestation of oestrus in cattle: a review. Applied Animal Behaviour Science, v. 70, n. 1, p. 1-16, 2000.

PINHO, T. G.; MUCCIOLO, R. G.; VERRESCHI, T. T. N. Níveis de progesterona no plasma sanguíneo durante o ciclo estral e início de prenhez em bovinos mestiços leiteiros (Bos taurus $x$ Bos indicus). Revista Brasileira de Reprodução Animal, v. 12, n. 1, p. 17-26, 1988.

RANDALL, D.; BURGGREN, W.; FRENCH, K. Animal physiology: mechanisms and adaptations. 5. ed. New York: W.H. Freeman and Co., 2002. 727 p.

REICHENBACH, H. D.; OLIVEIRA, M. A. L.; LIMA, P. F.; SANTOS-FILHO,A. S.;ANDRADE, J. C. O. Transferência e criopreservação de embriões bovinos. In: GONSALVES, P. B. D.; FIGUEIREDO, J. R.; FREITAS, V. J. F. Biotécnicas aplicadas à reprodução animal. 1. ed. São Paulo: Editora Varela, 2002. p. 127-177.

REMSEN L. G.; ROUSSEL J. D. Pregnancy rates relating to plasma progesterone levels in recipient heifers at day of transfer. Theriogenology, v. 18, p. 365-372, 1982.

RODRIGUES, S. M.; LEDOUX J. E.; SAPOLSKY R. M. The influence of stress hormones on fear circuitry. Annual Review Neuroscience, v. 32, p. 289-313, 2009.

SAS. Statistical analysis system user's guide: Stat., version 6.11. Cary: SAS Institute, 2005.

SHARF, B; WAX, L. E.; AIKEN, E.; SPIERS, D. E. Regional differences in sweat rate response of steers to short-term heat 
stress. International Journal Biometeorology, v. 52, n. 8, p. 725-732, 2008.

SILVA, R. G. Introdução à bioclimatologia animal. São Paulo: Nobel, 2000. 450 p.

SILVA, R. G.; MORAIS, D. A. E. F.; GUILHERMINO, M. M. Evaluation of thermal stress indexes for dairy cows in tropical regions. Revista Brasileira de Zootecnia, v. 36, n. 4, p. 1192 1198 (supl.), 2006.

STOKES, M. E.; DAVIS, C. S.; KOCH, G. G. Categorical data analysis using SAS system. 2. ed. Cary: 2000. 626 p.

TORRES-JÚNIOR, J. R. S.; PIRES, M. F. A.; SÁ, W. F.; FERREIRA, A. M.; VIANNA, J. H. M.; CAMARGO, L. S. A.; RAMOS, A. A.; FOLHADELLA I. M.; POLISSENI, J.; FREITAS, C.; CLEMENTE, C. A. A.; SÁ FILHO, M. F.; PAULA-LOPES, F.
F.; BARUSELLI, P. S. Effect of maternal heat-stress on follicular growth and oocyte competence in Bos indicus cattle. Theriogenology, v. 69, p. 155-166, 2008.

VASCONCELOS, J. L. M.; DEMÉTRIO, D. F. B.; SANTOS, R. M.; CHIARI, J. R.; RODRIGUES, C. A.; SÁ FILHO, O. G. Factors potencially affecting fertility of lactating dairy cows recipients. Theriogenology, v. 65, p. 192-200, 2006.

WILLARD, S. T.; LAY, D. C. JR.; FRIEND, T. H.; NEUENDORFF, D. A.; RANDEL, R. D. Plasma progesterone response following ACTH administration during mid-gestation in pregnant Brahman heifer. Theriogenology, v. 63, n. 4, p. 1061-1069, 2006.

WILTBANK, J. N. Effect of nutrition and other factors on the reproduction heifers. In: ANNUAL BEEF CATTLE SHORT COURSE, 26., 1983, Gainesville, FI, USA, Proceedings... Gainesville: University of Florida, 1983. p. 63-68.

Protocolado em: 18 jan. 2008. Aceito em: 2 mar. 2010. 